\title{
Histological Changes in Autoimmune Hepatitis with Graves' Disease: A Child Case Report
}

\author{
Mamiko Yamada ${ }^{1,2}$, Hironori Shibata ${ }^{1}$, Yohei Masugi ${ }^{1}$, Tomohiro Ishi ${ }^{1}$, Kaori Kameyama ${ }^{3}$, \\ Hirotoshi Ebinuma ${ }^{4}$ and Tomonobu Hasegawa ${ }^{1}$
}

\begin{abstract}
:
We herein report a child case of autoimmune hepatitis (AIH) accompanied with Graves' disease. Elevated aminotransferase levels were found in a 12-year-old Japanese girl with Graves' disease. In her first liver biopsy, necrosis and inflammation was limited to the centrilobular area, while the second biopsy showed different findings. Namely, portal injury newly appeared, including interface hepatitis, which represents the histological characteristics of AIH. As the histological findings at the onset of AIH do not always show typical findings, a re-biopsy is considered to be important in individuals suspected to have AIH. AIH should be included in the differential diagnosis of liver dysfunction in Graves' disease, even in children.
\end{abstract}

Key words: autoimmune hepatitis, interface hepatitis, Graves' disease, liver histology, pediatric case

(Intern Med 56: 2139-2143, 2017)

(DOI: 10.2169/internalmedicine.8417-16)

\section{Introduction}

Autoimmune hepatitis (AIH) is an immune-mediated liver disorder characterized serologically by high aminotransferase and immunoglobulin G levels, with the presence of autoantibodies, and histologically demosntrating interface hepatitis (1). Twenty percent of AIH patients have been reported to have other autoimmune diseases, such as thyroiditis, vitiligo, and type1 diabetes (2), and rarely Graves' disease (3). The definitive diagnosis of AIH depends on liver biopsy findings, including the presence of interface hepatitis with portal and periportal lymphoplasmacytic infiltrate (4). We herein report a child case of AIH accompanied with Graves' disease. It was interesting to note that the necrosis and inflammation was limited to the centilobular area at the first biopsy, however, these features were also noted in portal lesions at the second biopsy.

\section{Case Report}

A 12-year-old Japanese girl with a two-and-a-half-year re- mission of Graves' disease was found to have recurrence by routine following-up laboratory examinations; including increased free- $\mathrm{T}_{3}(6.62 \mathrm{pg} / \mathrm{mL})$ and free- $\mathrm{T}_{4}(1.84 \mathrm{ng} / \mathrm{dL})$, and low thyroid stimulating hormone (TSH) $(<0.01 \mu \mathrm{IU} / \mathrm{mL})$. She was treated with $5 \mathrm{mg}$ of methimazole (MMI) daily. After 2 months of MMI treatment, her aminotransferase levels were elevated (aspartate aminotransferase (AST) 344 IU/L, alanine aminotransferase (ALT) 494 IU/L) with an improving thyroid function. As her aminotransferase levels worsened despite the discontinuation of MMI, she was admitted to our institute. Her first diagnosis of Graves' disease had been at 5 years of age. The treatment was started using MMI (1 mg/kg/day) with gradual tapering. During this treatment, laboratory tests showed a consistently normal liver function. She had not taken any medication from 9 years and 9 months of age.

Her physical examination showed the following findings: height $147.4 \mathrm{~cm}$ (-1.1 SD), weight $36.6 \mathrm{~kg}$ (-1.2 SD), blood pressure $121 / 68 \mathrm{mmHg}$, pulse rate $88 / \mathrm{min}$ and temperature $36.8^{\circ} \mathrm{C}$. The thyroid gland was not enlarged and it showed a normal consistency. The liver was palpable $3 \mathrm{~cm}$ below the sternum, with a sharp edge and normal consistency. There

\footnotetext{
${ }^{1}$ Department of Pediatrics, Keio University School of Medicine, Japan, ${ }^{2}$ Tachikawa Hospital, Federation of National Public Service Personnel Mutual Aid Associations, Japan, ${ }^{3}$ Department of Pathology, Keio University School of Medicine, Japan and ${ }^{4}$ Division of Gastroenterology and Hepatology, Department of Internal Medicine, Keio University School of Medicine, Japan

Received: October 17, 2016; Accepted: January 11, 2017; Advance Publication by J-STAGE: August 1, 2017

Correspondence to Dr. Tomonobu Hasegawa, thaseg@a6.keio.jp and thaseg@sc.itc.keio.ac.jp
} 
Table. Patient's Clinical Laboratory Data on the First and the Second admission.

\begin{tabular}{|c|c|c|c|c|c|}
\hline Hematology & $1 \mathrm{st}$ & 2nd & Viral markers & $1 \mathrm{st}$ & 2nd \\
\hline $\mathrm{RBC}\left(\times 10^{4} / \mu \mathrm{L}\right)$ & 504 & 480 & IgM anti-HAV & negative $^{\dagger}$ & negative \\
\hline $\mathrm{Hb}(\mathrm{g} / \mathrm{dL})$ & 14.2 & 13.5 & HBsAg & negative & negative \\
\hline platelet $\left(\times 10^{4} / \mu \mathrm{L}\right)$ & 30.0 & 34.5 & Anti-HBc & negative $^{\dagger}$ & - \\
\hline $\mathrm{WBC}(/ \mu \mathrm{L})$ & 5,800 & 5,600 & Anti-HBs & negative & negative \\
\hline $\operatorname{ESR}(\mathrm{mm})$ & 10 & - & Anti-HCV & negative & negative \\
\hline Liver function tests & & & IgA-HEV & negative & - \\
\hline AST (IU/L) & 302 & 529 & VCA IgG & $\times 80^{\dagger}$ & - \\
\hline ALT (IU/L) & 433 & 833 & EBNA & $\times 20^{\dagger}$ & - \\
\hline Total bilirubin (mg/dL) & 1.1 & 2.1 & Immunology & & \\
\hline Direct bilirubin $(\mathrm{mg} / \mathrm{dL})$ & 0.2 & 1.0 & ANA & $1: 160^{\dagger}$ & $1: 640$ \\
\hline GGTP (IU/L) & 93 & 94 & SMA & negative $^{\dagger}$ & - \\
\hline ALP (IU/L) & 398 & 575 & anti-LKM1 & negative $^{\dagger}$ & - \\
\hline Albumin (g/dL) & 4.6 & 4.2 & AMA & negative & - \\
\hline PT $(\%)$ & 88 & 71 & $\operatorname{IgG}(\mathrm{mg} / \mathrm{dL})$ & 1,714 & 1,843 \\
\hline \multicolumn{6}{|l|}{ Thyroid function tests } \\
\hline TSH $(\mu \mathrm{IU} / \mathrm{mL})$ & 0.01 & 4.20 & & & \\
\hline free $T_{3}(p g / m L)$ & 6.9 & 3.0 & & & \\
\hline free $\mathrm{T}_{4}(\mathrm{ng} / \mathrm{dL})$ & 2.7 & 0.9 & & & \\
\hline TRAb (IU/L) & 0.4 & & & & \\
\hline
\end{tabular}

previous hospital data 4 days before the first admission.

ESR: erythrocyte sedimentation rate, AST: aspartate aminotransferase, ALT: alanine aminotransferase, GGTP: gamma-glutamyl transpeptidase, PT: prothrombin time, TSH: thyroid stimulating hormone, TRAb: TSH receptor antibody, ANA: antinuclear antibody, SMA: smooth muscle antibody, anti-LKM1: antibodies to liver/kidney microsome type1, AMA: antimitochondrial antibody, IgG: immunoglobuline G

were no skin eruptions. Laboratory tests showed elevated levels of aminotransferases (AST 302 IU/L, ALT 433 IU/L) and total bilirubin $(1.1 \mathrm{mg} / \mathrm{dL})$ being negative for $\mathrm{HBsAg}$, anti-HCV, IgM anti-HAV, and IgA-HEV. The thyroid function test was consistent with hyperthyroidism (free- $\mathrm{T}_{3} 6.9$ $\mathrm{pg} / \mathrm{mL}$, free-T $2.7 \mathrm{ng} / \mathrm{dL}$, and TSH $0.01 \mu \mathrm{IU} / \mathrm{mL}$ ). A druginduced lymphocyte stimulation test for MMI was negative. Serological tests for anti-smooth muscle antibody (SM), anti-liver/kidney microsomal type1 (anti-LKM) and antimitochondrial antibody (AMA) were negative, whereas antinuclear antibody (ANA) was positive (1:160). The serum level of $\operatorname{IgG}(1,714 \mathrm{mg} / \mathrm{dL})$ was above the normal range (Table).

Liver biopsy was performed on the 8th day after admission, but a histological diagnosis could not be made. The biopsy specimen showed a necroinflammatory reaction with the infiltration of lymphocytes, plasma cells and eosinophils exclusively at the centrilobular area while no inflammatory cell infiltrate at the portal or periportal area, which was uncommon as liver disease except for drug-induced liver injury (Fig. 1A and B), which was not typical for primary sclerosing cholangitis and primary biliary cirrhosis. At this moment, there were some possible causes of this liver injury; drug-induced hepatotoxicity, autoimmune hepatitis, and Graves' disease itself. The low score (+2 points) for the diagnostic criteria of drug induced liver injury was less likely to be diagnosed with it (5). Drug-induced hepatotoxicity was also quite unlikely considering that hepatocanalicular cholestasis was identified in the histological findings (6). The his- tological features were inconsistent with $\mathrm{AIH}$; there were no findings of interface hepatitis or lymphoplasma cell infiltration. The score of the revised original pretreatment scoring system of the International Autoimmune Hepatitis Group was 14 (Supplementary material 1) (7) and the simplified diagnostic criteria for AIH (Supplementary material 2) (8) was 5 , both scores did not meet the criteria of the definite diagnosis of AIH. The possibility that Graves' disease itself was not excluded completely, but it could not be definitively diagnosed because of the nonspecific histological findings of liver injury caused by Graves' disease, such as hepatic necrosis, fatty deposition, hepatatrophia and liver congestion $(9,10)$. Although we could not define the cause of liver injury, the liver and thyroid function had improved without any treatment. She thus discharged from our institute.

One month later, as the aminotransferase levels became worse (AST 809 IU/L, ALT 1,220 IU/L), she was readmitted (Table). The second liver biopsy showed quite different findings from those of the first one: definite interface hepatitis with lymphoplasmacytic inflammation at the portal and periportal area in addition to increased inflammation at the centrilobular area (Fig. 1C and D). She was diagnosed to have AIH by these histological findings and the scoring system of AIH. There were findings of interface hepatitis, and lymphoplasma cell infiltration into the portal area, but no rosette formation, or emperipolesis. The score of the revised original pretreatment scoring system of the International Autoimmune Hepatitis Group was 21 (Supplementary material 1) (7) and the simplified diagnostic criteria for $\mathrm{AIH}$ 


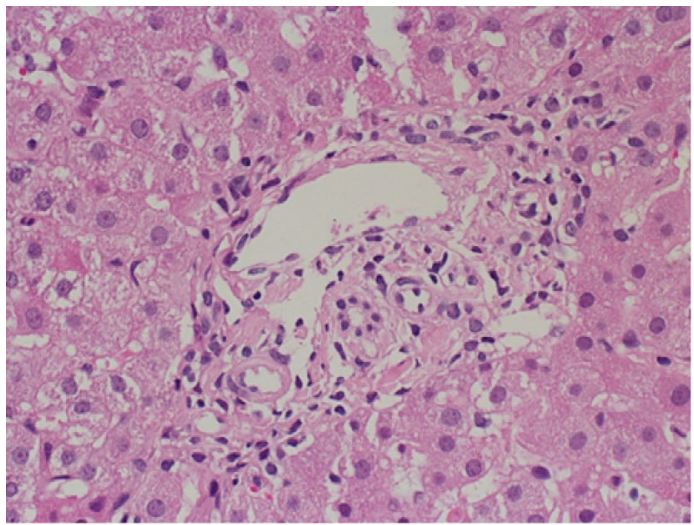

(A)

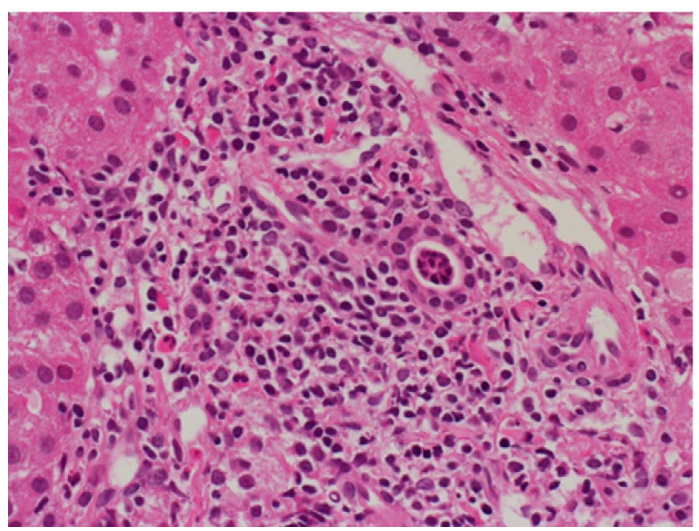

(C)

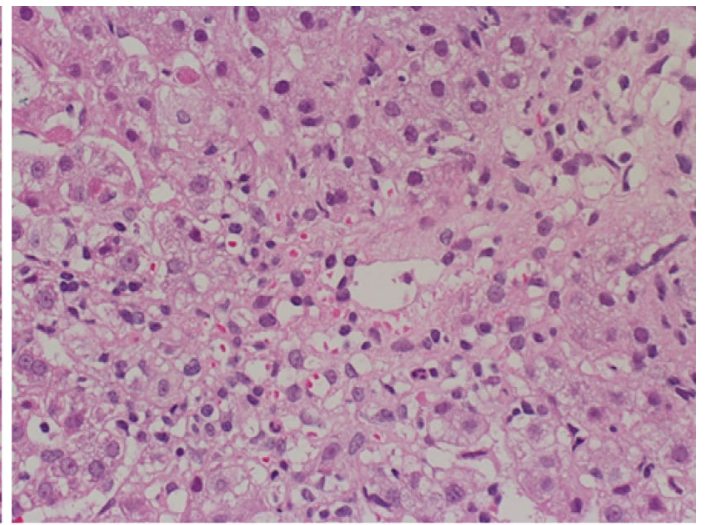

(B)

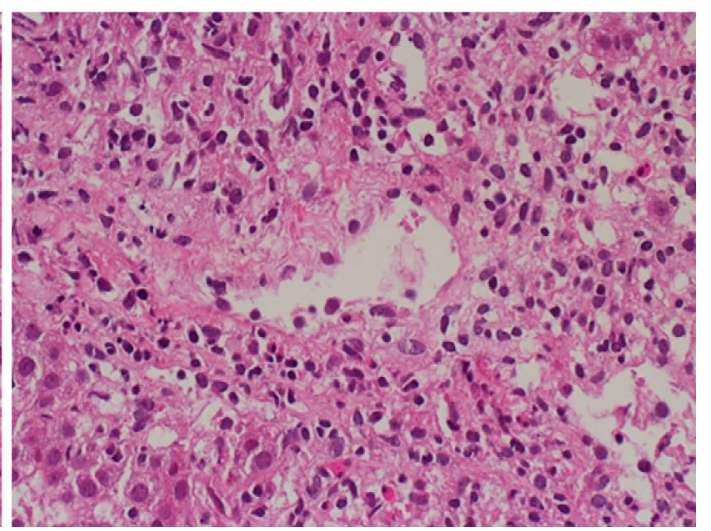

(D)

Figure 1. The histological findings of liver biopsies. A and B were on the first biopsy, $C$ and $D$ were on the second biopsy. (A) Inflammatry cells were scarcely seen at the portal or periportal area at the first biopsy. (B) Moderate necroinflammatory reaction with infiltration of lymphocytes, plasma cells and eosinophils was seen at the centilobular area at the first biopsy. (C) An evident necroinflammatory reaction with lymphoplasmacytic infiltration was seen at the portal and periportal area. Compared with the first biopsy, the marked portal inflammation was revealed. (D) Mild to moderate necroinflammatory reaction was also seen at the centliobular area at the second biopsy.

(Supplementary material 2) (8) was 7 , both scores met the criteria for the definite diagnosis of AIH. At this point, Graves' disease itself was eliminated as the cause of liver injury, considering the increasing aminotransferases under stable thyroid function. We started the patient on prednisone and azathioprine therapy, the first dosage of each was 30 $\mathrm{mg} /$ day and $25 \mathrm{mg} / \mathrm{day}$, respectively, and then the liver function rapidly improved. The clinical remission continued while gradually decreasing the dosage of prednisone by 5 $\mathrm{mg}$ about every two weeks. At present, the patient takes 5 $\mathrm{mg}$ of prednisone and $25 \mathrm{mg}$ of azathioprine. Her liver function has been within the reference range (Fig. 2).

\section{Discussion}

The characteristics of AIH include the presence of autoantibodies, hypergammmaglobulinemia, and histological features with interface hepatitis (11). In this case, the diagnosis of AIH was made based on the following reasons: 1) this case met the criteria of AIH; the revised original pretreatment scoring system of the International Autoimmune Hepa- titis Group (Supplementary material 1) (7), the simplified diagnostic criteria for AIH (Supplementary material 2) (8) and the guidelines of AIH in Japan (4), 2) high level of IgG and ANA, 3) the findings of the second liver biopsy were compatible with $\mathrm{AIH}, 4)$ the exclusion of other causes of liver injury as Graves' disease itself and drug-induced. Considering the worsening liver function with a stable thyroid function, Graves' disease itself was not the cause of the liver dysfunction. Taking the clinical course into account, medication was eliminated as the cause of liver dysfunction; aminotransferase was getting worse after discontinuation of MMI and the patient did not take any other medication.

The course of this patient suggested two important clinical issues. First, interface hepatitis was apparent at the later stage of AIH. At the early stage of AIH, the main lesion of necrosis and inflammation was limited to the centrilobular area. Second, for child patients with Graves' disease, AIH might be one of the causes of liver dysfunction. This case clearly demonstrated that necrosis and inflammation in the portal area appeared following the occurrence of centrilobular necrosis $(12,13)$. In a past report, AIH patients with cen- 


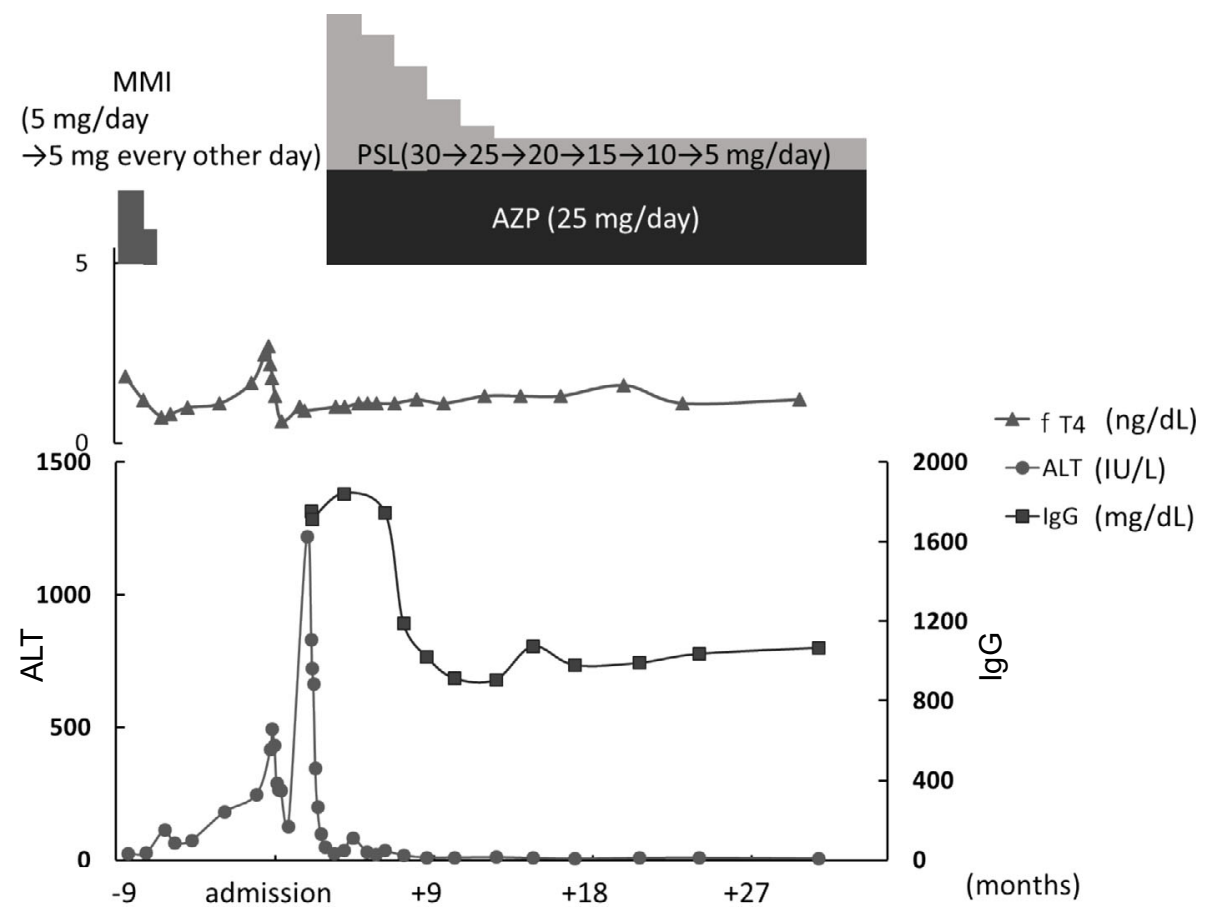

Figure 2. Clinical course of the patient. MMI: methimazole, PSL: prednisone, AZP: azathioprine

trilobular necrosis are more likely to have an acute onset of disease than those without (14). Our case showed the histological change of AIH to be consistent with the findings of two previous reports $(15,16)$. There were common features in the initial biopsy, while inflammation was not found in the portal area, but instead in the centrilobular area, and the second biopsy showed a worsening of pericentral inflammation and the appearance of portal inflammation. These histological changes may therefore represent the natural course of AIH.

For child patients with Graves' disease, physicians should therefore keep the possibility of AIH in mind as one of potential causes of liver dysfunction. The available data show that AIH has two age peaks, one is at around 70 years of age while the another is at from $7-11$ years of age $(17,18)$. The clinical characteristics of both groups are also quite similar, such as a female dominance and the proportion of comorbidity with immune-mediated disease $(2,19,20)$. In adults, the most common associated disease is autoimmune thyroiditis, while in children, it is inflammatory bowel disease (15). The four past case reports of patients with both AIH and Graves' disease were all women over 20 years of age (21-24). Except for one case, in which a liver biopsy was not done, all cases had the typical histological findings of AIH. In the case of liver dysfunction with Graves's disease, it is therefore important to keep the possibility of AIH in mind, even in children.

In this case, the possibility of drug-induced AIH was quite unlikely since ALT decreased spontaneously to within the normal range during the 1st hospitalization.

In conclusion, this patient with Graves's disease had liver dysfunction with atypical histological findings of AIH in the first biopsy. Necrosis and inflammation may be limited to the centrilobular area in the early stage of AIH, and thereafter expand to the portal area as liver dysfunction progresses. For child patients with Graves' disease, AIH may thus be the cause of liver dysfunction. When a liver biopsy shows centilobular necrosis and inflammation in a patient suspected of having $\mathrm{AIH}$, then a second liver biopsy is warranted.

The authors state that they have no Conflict of Interest (COI).

Acknowledgement

We thank Prof. Takao Takahashi for fruitful discussions.

\section{References}

1. Mieli-Vergani G, Vergani D. Autoimmune hepatitis. Nat Rev Gastroenterol Hepatol 8: 320-329, 2011.

2. Gregorio GV, Portmann B, Reid F, et al. Autoimmune hepatitis in childhood: a 20-year experience. Hepatology 25: 541-547, 1997.

3. Inoue K, Okajima T, Tanaka E, et al. A case of Graves' disease associated hepatitis and mixed connective tissue disease. Endocr J 46: 173-177, 1999.

4. Onji M, Zeniya M, Yamamoto K, Tsubouchi H. Autoimmune hepatitis: diagnosis and treatment guide in Japan, 2013. Hepatol Res 44: 368-370, 2014.

5. Takikawa H, Onji M. A proposal of the diagnostic scale of druginduced liver injury. Hepatol Res 32: 250-251, 2005.

6. Schmidt G, Börsch G, Müller KM, Wegener M. Methimazoleassociated cholestatic liver injury: case report and brief literature review. Hepatogastroenterology 33: 144-146, 1986.

7. Alvarez F, Berg PA, Bianchi FB, et al. International Autoimmune Hepatitis Group Report: review of criteria for diagnosis of autoimmune hepatitis. J Hepatol 31: 929-938, 1999.

8. Hennes EM, Zeniya M, Czaja AJ, et al. Simplified criteria for the diagnosis of autoimmune hepatitis. Hepatology 48: 169-176, 2008.

9. Beaver DC, Pemberton J. The pathologic anatomy of the liver in exophthalmic goiter. Ann Intern Med 7: 687-694, 1933.

10. Movitt ER, Gerstl B, Davis AE. Needle liver biopsy in thyrotoxi- 
cosis. AMA Arch Intern Med 91: 729-739, 1953.

11. Guindi M. Histology of autoimmune hepatitis and its variants. Clin Liver Dis 14: 577-590, 2010.

12. Manns MP, Strassburg CP. Autoimmune hepatitis: clinical challenges. Gastroenterology 120: 1502-1517, 2001.

13. Stravitz RT, Lefkowitch JH, Fontana RJ, et al. Autoimmune acute liver failure: proposed clinical and histological criteria. Hepatology 53: 517-526, 2011.

14. Hofer H, Oesterreicher C, Wrba F, Ferenci P, Penner E. Centrilobular necrosis in autoimmune hepatitis: a histological feature associated with acute clinical presentation. J Clin Pathol 59: 246249, 2006.

15. Pratt DS, Fawaz KA, Rabson A, Dellelis R, Kaplan MM. A novel histological lesion in glucocorticoid-responsive chronic hepatitis. Gastroenterology 113: 664-668, 1997.

16. Singh R, Nair S, Farr G, Mason A, Perrillo R. Acute autoimmune hepatitis presenting with centrizonal liver disease: case report and review of the literature. Am J Gastroenterol 97: 2670-2673, 2002.

17. Gronbaek L, Vilstrup H, Jepsen P. Autoimmune hepatitis in Denmark: incidence, prevalence, prognosis, and causes of death. A nationwide registry-based cohort study. J Hepatol 60: 612-617, 2014.

18. Floreani A, Liberal R, Vergani D, Mieli-Vergani G. Autoimmune hepatitis: contrasts and comparisons in children and adults - a comprehensive review. J Autoimmun 46: 7-16, 2013.
19. Dehghani SM, Haghighat M, Imanieh $\mathrm{MH}$, et al. Autoimmune hepatitis in children: experiences in a tertiary center. Iran J Pediatr 23: 302-308, 2013.

20. Gregorio GV, Portmann B, Karani J, et al. Autoimmune hepatitis/ sclerosing cholangitis overlap syndrome in childhood: a 16-year prospective study. Hepatology 33: 544-553, 2001.

21. Takamoto $S$, Tahara $M$, Yofune $H$, et al. A case of autoimmune hepatitis with Graces' disease. Shounika Rinsho (Jpn J Pediatr) 52: 1841-1845, 1999 (in Japanese, Abstract in English).

22. Abe K, Saito H, Takahashi A, et al. A case of acute hepatitis associated with Basedow's disease. Nihon Shokakibyo Gakkai Zasshi (J Jpn Soc Gostroenterol) 104: 52-56, 2007 (in Japanese, Abstract in English).

23. Cui B, Abe M, Hidata S, et al. Autoimmune hepatitis associated with Graves' disease. Intern Med 42: 331-335, 2003.

24. Jhee JH, Kim HJ, Kang W, Kim S, Kim do Y. A case of autoimmune hepatitis combined with Graves' disease. Korean J Gastroenterol 65: 48-51, 2015.

The Internal Medicine is an Open Access article distributed under the Creative Commons Attribution-NonCommercial-NoDerivatives 4.0 International License. To view the details of this license, please visit (https://creativecommons.org/licenses/ by-nc-nd/4.0/).

(C) 2017 The Japanese Society of Internal Medicine Intern Med 56: 2139-2143, 2017 